\title{
On the sources of static plane symmetric vacuum space-times
}

\author{
M.F.A. da Silva * and Anzhong Wang ${ }^{\dagger}$ \\ Departamento de Física Teórica, Universidade do Estado do Rio de Janeiro, \\ Rua São Francisco Xavier 524, Maracanã, 20550-013 Rio de Janeiro - RJ, Brazil \\ and \\ Observatório Nacional - CNPq, Rua General José Cristino 77, \\ São Cristóvão, 20921-400 Rio de Janeiro - RJ, Brazil \\ N. O. Santos ${ }^{\ddagger}$ \\ Departamento de Astrofísica, Observatório Nacional - CNPq, \\ Rua General José Cristino 77, São Cristóvão, 20921-400 Rio de Janeiro - RJ, Brazil \\ and \\ Departamento de Física Teórica, Universidade do Estado do Rio de Janeiro, \\ Rua São Francisco Xavier 524, Maracanã, 20550-013 Rio de Janeiro - RJ, Brazil
}

October 15, 2018

\footnotetext{
*e-mail address: mfas@symbcomp.uerj.br

$\dagger$ e-mail address: wang@symbcomp.uerj.br

$\ddagger$ e-mail address: nos@on.br
} 


\begin{abstract}
The static vacuum plane spacetimes are considered, which have two non-trivial solutions: The Taub solution and the Rindler solution. Imposed reflection symmetry, we find that the source for the Taub solution does not satisfy any energy conditions, which is consistent with previous studies, while the source for the Rindler solution satisfies the weak and strong energy conditions (but not the dominant one). It is argued that the counterpart of the Einstein theory to the gravitational field of a massive Newtonian plane should be described by the Rindler solution, which represents also a uniform gravitational field.
\end{abstract}

PACS numbers: 04.20Jb, 04.40.+c. 
It is well-known that the unique solution of vacuum static plane symmetric space-time with non-null curvature is the Taub plane solution [1], which in the usual plane symmetric coordinates, $x^{\mu}=\{T, Z, X, Y\}(\mu=0,1,2,3)$, is given by

$$
d s^{2}=\frac{1}{Z^{2 / 3}} d T^{2}-d Z^{2}-Z^{4 / 3}\left(d X^{2}+d Y^{2}\right) .
$$

As shown by Taub himself, the space-time is asymptotically flat as $|Z| \rightarrow+\infty$ and singular at $Z=0$. It is generally believed that the singularity should be replaced by a regular source for more realistic models. It is this belief that attracted much of attention in finding sources for the Taub solution [2]. In particular, Dolgov and Khriplovich [3] showed that static space-times with reflection plane symmetry are not free of space-time singularity, provided that the energy density of the sources is positive. This implies that the sources of Taub's solution have negative energy density, if we require that the spacetime be static, reflection symmetric, and free of space-time singularity.

On the other hand, from the work of Vilenkin [4] and Novotný et al [5] we can see that the Newtonian limit of such space-times exists. As a matter of fact, it takes the form

$$
d s^{2}=(1+8 \pi \rho|Z|) d T^{2}-(1-8 \pi \rho|Z|)\left(d Z^{2}+d X^{2}+d Y^{2}\right),
$$

which satisfies the linearized Einstein's field equations $\square \Psi_{\mu \nu}=8 \pi \rho \delta(Z) \delta_{\mu}{ }^{T} \delta_{\nu}{ }^{T}$, where $\delta(Z)$ denotes the Dirac delta function, and $\rho$ the surface energy density 
of the plane $Z=0$. The results obtained in [2, 3] and the ones obtained in [䍘, 5] seemingly contradict: There exists Newtonian limit but does not exist exact solutions to the Einstein field equations.

In this Letter, we shall show that there is no contradiction. In fact, exact solutions with positive mass indeed exist but the space-time outside sources is not Taub's, rather than that of Rindler [6], which represents an uniform gravitational field. Recall that the gravitational field of a massive plane in Newtonian theory is also uniform!

To show the above, let us start with the general form of the metric for the static plane symmetric space-times [1]

$$
d s^{2}=f(z)\left(d t^{2}-d z^{2}\right)-g(z)\left(d x^{2}+d y^{2}\right)
$$

where $\left\{x^{\mu}\right\} \equiv\{t, z, x, y\}$ is another set of plane symmetric coordinates.

The non-vanishing components of the Einstein tensor for the above metric are given by

$$
\begin{aligned}
& G_{00}=\frac{1}{4 f g^{2}}\left(f g^{2}-4 f g g^{\prime \prime}+2 g f^{\prime} g^{\prime}\right), \\
& G_{11}=\frac{1}{4 f g^{2}}\left(2 g f^{\prime}+f g^{\prime}\right) g^{\prime}, \\
& G_{22}=G_{33}=\frac{1}{4 g f^{3}}\left(2 f g^{2} f^{\prime \prime}-2 g^{2} f^{\prime 2}+2 g f^{2} g^{\prime \prime}-f^{2} g^{\prime 2}\right),
\end{aligned}
$$

where a prime denotes the ordinary differentiation with respect to $z$.

¿From the expression of $G_{11}$, we can see that the vacuum solutions of the 
Einstein's field equations can be divided into three different cases:

i) $2 g f^{\prime}+f g^{\prime}=0, \quad g^{\prime} \neq 0$; ii) $2 g f^{\prime}+f g^{\prime} \neq 0, \quad g^{\prime}=0$; iii) $f^{\prime}=0=g^{\prime}$.

In Case i), using equations (14) it is easy to show that the general Einstein vacuum solution is given by

$$
f(z)=\frac{\alpha^{2}}{\sqrt{z+\gamma}}, \quad g(z)=\beta^{2}(z+\gamma)
$$

where $\alpha, \beta$ and $\gamma$ are integration constants. Setting

$T=\left(\frac{4 \alpha^{4}}{3}\right)^{1 / 3} t, \quad Z=\frac{4 \alpha}{3}(z+\gamma)^{3 / 4}, X=\beta\left(\frac{3}{4 \alpha}\right)^{2 / 3} x, \quad Y=\beta\left(\frac{3}{4 \alpha}\right)^{2 / 3} y$,

the corresponding solution will take the same form as that given by equation (11). Therefore, Case i) gives exactly the Taub solution.

In Case ii), it can be shown that the Einstein vacuum equations have the general solution

$$
f(z)=e^{a z+b}, \quad g(z)=c^{2},
$$

where $a, b$ and $c$ are arbitrary constants. Introducing the new coordinates

$$
T=\frac{a}{2} t, \quad Z=\frac{2}{a} e^{(a z+b) / 2}, \quad X=c x, \quad Y=c y,
$$

the corresponding metric will take the form

$$
d s^{2}=Z^{2} d T^{2}-d Z^{2}-\left(d X^{2}+d Y^{2}\right)
$$

but this is the Rindler solution [6], which represents an uniform gravitational field, and the corresponding Riemann tensor is identically zero. 
In Case iii), the vacuum Einstein field equations have the trivial solution, $f=$ Const. and $g=$ Const. Clearly, this exactly corresponds to the Minkowski space-time.

Therefore, in general, the static Einstein vacuum field equations with plane symmetry have two non-trivial classes of solutions. To find sources for these two classes of solutions, we shall model the sources as infinitely thin planes. The main reason for doing so is because in this case the Newtonian theory has an unique solution. Moreover, as showed in [4, 5], its Newtonian limit also exists. To construct such sources, we shall use the ansatz,

$$
z \rightarrow|z|
$$

in the solutions (5) and (6), respectively. Such a substitution is physically equivalent first to cut a space-time into two regions, $z>0$ and $z<0$, and then join the region $z>0$ with a copy of it, so that the resulted space-time has a reflection symmetry with respect to the surface $z=0$. Clearly, this is possible only when a matter shell appears on the surface, which serves as a mirror of the gravitational field.

The form of the matter shell can be calculated using Taub's formulae [0]. Following Taub \&, we first introduce a surface tensor $b_{\mu \nu}$ via the relations

$$
\left[g_{\mu \nu, \lambda}\right]^{-}=\left.g_{\mu \nu, \lambda}^{+}\right|_{z=0^{+}}-\left.g_{\mu \nu, \lambda}^{-}\right|_{z=0^{-}}=\eta_{\lambda} b_{\mu \nu}
$$

\footnotetext{
${ }^{1}$ The formulae given by Taub are equivalent to those of Israel [8], when the matching hypersurface is spacelike or timelike, as shown by Taub himself [7] (See also [10]).
} 
where $\eta_{\lambda}$ is the normal vector to the hypersurface $z=0$, and is given by $\eta_{\lambda}=$ $\delta^{z}{ }_{\lambda} \cdot g_{\mu \nu}^{+}\left(g_{\mu \nu}^{-}\right)$are the metric coefficients given in the region $z>0(z<0)$. Once $b_{\mu \nu}$ is known, the surface energy-momentum tensor $\tau_{\mu \nu}$ can be read off from the expression

$$
\tau_{\mu \nu}=\frac{1}{16 \pi}\left\{b\left(\eta g_{\mu \nu}-\eta_{\mu} \eta_{\nu}\right)+\eta_{\lambda}\left(\eta_{\mu} b_{\nu}{ }^{\lambda}+\eta_{\nu} b_{\mu}{ }^{\lambda}\right)-\left(\eta b_{\mu \nu}+\eta_{\lambda} \eta_{\delta} b^{\lambda \delta} g_{\mu \nu}\right)\right\},
$$

where $\eta \equiv \eta_{\lambda} \eta^{\lambda}$ and $b \equiv b_{\lambda}{ }^{\lambda}$. In terms of $\tau_{\mu \nu}$ the energy-momentum tensor $T_{\mu \nu}$ of the four-dimensional space-time takes the form $T_{\mu \nu}=T_{\mu \nu}^{+} H(z)+$ $T_{\mu \nu}^{-} H(z)+\tau_{\mu \nu} \delta(z)$, where $H(z)$ denotes the Heaviside function, which is one for $z \geq 0$ and zero for $z<0$. $T_{\mu \nu}^{ \pm}$are the energy-momentum tensor calculated respectively in the regions $z>0$ and $z<0$. In the present case, since the space-time is vacuum in these two regions, we have $T_{\mu \nu}^{ \pm}=0$.

For the general metric (3), it can be shown that $\tau_{\mu \nu}$ can be written as

$$
\tau_{\mu \nu}=\rho u_{\mu} u_{\nu}+p\left(x_{\mu} x_{\nu}+y_{\mu} y_{\nu}\right)
$$

where

$$
\rho=-\frac{\left[g^{\prime}\right]^{-}}{8 \pi f g}, \quad p=\frac{1}{16 \pi f^{2} g}\left[(f g)^{\prime}\right]^{-},
$$

and

$$
u_{\mu} \equiv \sqrt{f} \delta_{\mu}{ }^{t}, \quad x_{\mu} \equiv \sqrt{g} \delta_{\mu}{ }^{x}, \quad y_{\mu} \equiv \sqrt{g} \delta_{\mu}{ }^{y},
$$

with $\left.[G]^{-} \equiv G^{+}\right|_{z=0^{+}}-\left.G^{-}\right|_{z=0^{-}}$. Clearly, $\rho$ represents the surface energy 
density of the shell, and $p$ the pressure in the $d x$ and $d y$ directions, measured by observers who are at rest with respect to the shell.

Substituting equation (17) into equation (5), we find

$$
f(z)=\frac{\alpha^{2}}{\sqrt{|z|+\gamma}}, \quad g(z)=\beta^{2}(|z|+\gamma) .
$$

As mentioned previously, Taub's solution is singular on $z+\gamma=0$. To avoid this singularity in the solution (13), we require $\gamma>0$. Clearly, the spacetime in the region $z>0$ is locally isometric to Taub's with $Z>Z_{0}$, where $Z_{0} \equiv$ $(4 \alpha / 3) \gamma^{3 / 4}$, while in the region $z<0$, it is a copy of the spacetime of the region $z>0$. On the hypersurface $z=0$, the surface energy-momentum tensor is given by equation (10) with $\rho$ and $p$ now being given by

$$
\rho=-4 p=-\frac{1}{4 \pi \alpha^{2} \sqrt{\gamma}} .
$$

Clearly, now the surface energy density of the shell is negative and does not satisfy any of the three energy conditions, weak, dominant and strong [9]. This is consistent with the results obtained in [2, 3]. It is interesting to note that $\rho$ and $p$ both depend on $\alpha^{2} \sqrt{\gamma}$, which characterizes the strength of the energy density per unit area of the shell. This is understandable, if we recall that its Newtonian counterpart also has one independent parameter, although sometimes people tend to believe that Taub's solution essentially has no physically independent free parameters. 
On the other hand, substituting equation (7) into equation (6) we find

$$
f(z)=e^{a|z|+b}, \quad g(z)=c^{2} .
$$

Then, inserting these expressions into equation (11) we obtain

$$
\rho=0, \quad p=\frac{a e^{-b}}{8 \pi}
$$

Thus, in this case the energy density of the shell measured by those observers who are comoving with the shell is zero, while the pressure in the $d x$ and $d y$ directions in general is not. This is a little bit strange. The reason for it is that the shell does not satisfy the dominant energy condition, as one can easily show from equation (16). However, it does satisfy the weak and strong energy conditions, provided $a>0$. Moreover, only the comoving observers measure zero surface energy density. Other observers usually do not. For example, observers with the four-velocity $v_{\mu}=\sqrt{\left(1+\delta^{2}\right) f} \delta_{\mu}{ }^{t}+\delta \sqrt{g} \delta_{\mu}{ }^{x}$ will measured the density as $\rho_{v}=T_{\mu \nu} v^{\mu} v^{\nu}=\delta^{2} p \geq 0$, where $\delta$ is an arbitrary constant. It should be noted that our results do not contradict with the ones obtained in [3]. In fact, the function $u$ defined there is a constant in the present case, a case that is excluded in [3].

As showed above, the most general non-trivial static plane vacuum solutions are the Taub solution and the Rindler solution, and the sources of the Taub solution always have negative energy density. Therefore, the unique solution that corresponds to the Newtonian limit (2) is the solution given 
by equation (15). The space-time outside the source represents an uniform gravitational field, and is locally isometric to the Rindler solution [6]. The coordinate transformations that bring the solution of (15) (for $p$ being very small) to the one of equation (2) are complicate, and we have not found them, yet.

It is well-known that Rindler space-time has a non-trivial topology. In particular, it has event horizons. Thus, it would be very interesting to study the global structure of the solution (15). To do this, let us first consider the following coordinate transformations

$$
\begin{aligned}
& \bar{T}=\frac{2}{a} e^{a z+b / 2} \sinh \frac{a t}{2}, \quad \bar{Z}=\frac{2}{a} e^{a z / 2} \cosh \frac{a t}{2}, \\
& \bar{X}=c x, \quad \bar{Y}=c y,(z \geq 0) .
\end{aligned}
$$

Then we find that the metric takes exactly the Minkowski form $d s^{2}=d \bar{T}^{2}-$ $d \bar{Z}^{2}-d \bar{X}^{2}-d \bar{Y}^{2}$ in this region. From equation (17) we obtain

$$
\bar{Z}^{2}-\bar{T}^{2}=4 / a^{2}, \quad(z \geq 0) .
$$

Equations (17) and (18) show the mapping between the $(t, z)$-plane and the $(\bar{T}, \bar{Z})$-plane. In particular, the half plane $z>0$ is mapped to region $I$ where $\bar{Z}>\left(4 a^{-2} e^{a z+b}+\bar{T}^{2}\right)^{1 / 2}$ [cf. Fig. 1], while the hypersurface $z=0$ is mapped to one branch of the hyperbole $\bar{Z}^{2}-\bar{T}^{2}=4 a^{-2} e^{b}(\bar{Z}>0)$. Region $I^{\prime}\left[\right.$ where $\left.\bar{Z}<-\left(4 a^{-2} e^{a z+b}+\bar{T}^{2}\right)^{1 / 2}\right]$ is an extended region, while the region 
$-\left(4 a^{-2} e^{a z+b}+\bar{T}^{2}\right)^{1 / 2}<\bar{Z}<\left(4 a^{-2} e^{a z+b}+\bar{T}^{2}\right)^{1 / 2}$ corresponds to $z<0$ and does not belong to the space-time described by equation (15). Note that the hypersurfaces $\bar{T}^{2}=\bar{Z}^{2}$ represent the event horizons of the Rindler space-time [6], which is excluded from the space-time.

On the other hand, in region $z \leq 0$, if we make the same coordinate transformations (17) but with $z$ being replaced by $-z$, the corresponding solution (15) also takes the Minkowski form but now with

$$
\tilde{Z}^{2}-\tilde{T}^{2}=\frac{4}{a^{2}} e^{b}, \quad(z \leq 0) .
$$

Thus, the global structure of this region is similar to that with $z \geq 0$. In particular, the half plane $z<0$ is mapped to region $I I$ where $\tilde{Z}>$ $\left(4 a^{-2} e^{a z+b}+\tilde{T}^{2}\right)^{1 / 2}$ [cf. Fig. 2], while the hypersurface $z=0$ is mapped to the branch of the hyperbole $\tilde{Z}^{2}-\tilde{T}^{2}=4 a^{-2} e^{b}, \quad \tilde{Z}>0$. Region $I I^{\prime}$ where $\tilde{Z} \leq-\left(4 a^{-2} e^{-a z+b}+\tilde{T}^{2}\right)^{1 / 2}$ is an extended region in the Minkowski coordinates $\{\tilde{T}, \tilde{Z}, \tilde{X}, \tilde{Y}\}$. The global structure of the whole space-time is illustrated by Fig. 2. From there we can see that there essentially exist two plane shells $\overline{a b}$ and $\overline{c d}$. Each of the two shells connected two flat regions, in which the gravitational field is uniform, and these two regions are geodesically complete and free of any event horizons. 


\section{Acknowledgment}

The authors gratefully acknowledge financial assistance from CNPq.

\section{References}

[1] A.H. Taub, Ann. Math. 53, 472 (1951).

[2] B.W. Bonnor, Gen. Rel. Grav. 24, 551 (1992); B. Jensen and J. Kucĕra, Phys. Lett. A195, 111 (1994); M.L. Bedran, M.O. Calvão, F.M. Paiva, and I.D. Soares, Phys. Rev. D55, 3431 (1997), and references therein.

[3] A.D. Dolgov, and I.B. Khriplovich, Gen. Rel. Grav. 21, 13 (1989).

[4] A. Vilenkin, Phys. Rev. D23, 852 (1981).

[5] J. Novotný, J. Kucĕra, and J. Horský, Gen. Rel. Grav. 19, 1195 (1987).

[6] W. Rindler, "Essential Relativity", Revised Second Edition (SpringerVerlag, Berlin, 1977), pp.156-164.

[7] A.H. Taub, J. Math. Phys. 21, 1423 (1980).

[8] W. Israel, Nuovo Cimento, B44, 1 (1966); ibib. B48, 463(E) (1967).

[9] S.W. Hawking and G.F.R. Ellis, "The Large Scale Structure of Spacetime," (Cambridge University Press, Cambridge, 1973), pp.88-96. 
[10] A.Z. Wang, M.F.A da Silva, and N.O. Santos, "On parameters of the Levi-Civita solution," to appear in Class. Quantum Grav. (1997). 


\section{Figure Captions}

Fig. 1 The spacetime project for the region $z \geq 0$ in the $(\bar{T}, \bar{Z})$-plane for the

solution (15). Region $I$ corresponds to $z>0$, while region $I^{\prime}$ is an extended region in the Minkowski-like coordinates, $\bar{T}, \bar{Z}, \bar{X}$ and $\bar{Y}$.

Fig. 2 The corresponding Penrose diagram for the solution (15). $\overline{a b}$ and $\overline{c d}$ represent two thin matter shells, each of which connects two asymptotically flat regions. Therefore, each of the two diamonds represents a geodesically complete spacetime. 
This figure "fig1-1.png" is available in "png" format from: http://arxiv.org/ps/gr-qc/9706071v1 
This figure "fig1-2.png" is available in "png" format from: http://arxiv.org/ps/gr-qc/9706071v1 